\title{
Reliable Video Broadcasting for the E-Learning Environment
}

\author{
Mahmood Abdul Hakeem Abbood ${ }^{1}$, Prof. Dr. Nasser Nafe'a Khamees ${ }^{2}$ \\ Master Students, The informatics Institute for Postgraduate Studies, \\ Iraqi Commission for Computers and Information, Baghdad, $\mathrm{Iraq}^{1}$ \\ AL Nahrain University-College of Information Engineering, Baghdad, Iraq ${ }^{2}$
}

\begin{abstract}
This paper deals with how to broadcast the video over the e-learning network with achieving the reliability, low encoding bitrate, and high-quality video. The reliability in this work is achieved based on using h.264 encoder and incorporating the controller that adaptive the video output bitrate. The combination of two protocols (OSPF and PIM) will be used for routing and transmitting the packets over the network. The compression on the source video is optimized by using optimal configuration of h.264 codec parameters, while the bitrate is matched with the channel by incorporating subsystem as an adapter between the encoder and the channel. The test and measurements of video compression were achieved using JM 19.0. The broadcasting quality is measured by the networks QoS measurements; delay, jitter, and throughput that show the fidelity of the overall proposed model.
\end{abstract}

Keywords: H.264, GOP, rate controller, QP, OSPF, QoS

\section{INTRODUCTION}

In nowadays the video is one of the most important aspects. It can be defined as a computer-based interactive communications process that contains the video conferencing, IPTV, and e-learning applications[1]. Due to the rapid growth of multimedia communication, multimedia standards have received much attention during the last decade. So because the video consumes the higher bitrate has emerged as a major research and development area. In particular, the multimedia network opens a wide range of possibilities by combining different types of network protocols like multicast protocols with routing protocols or combination between two routing protocols etc. [2][3]. So the applications that are deals with video are Video-conferencing it is a technology that allows users in different locations to hold faceto-face meetings without having to move to a single location together. This technology is particularly convenient for business users in different cities or even different countries because it saves the time, expense and hassle associated with business travel. another application IPTV is the process of transmitting and broadcasting television programs through the Internet using Internet Protocol (IP). IPTV gives dynamic features to the user to improve the user experience compared to a traditional television transmission such as radio frequency broadcast, satellite broadcast and/or cable television while video for e-learning it is the process to transmit the lecturer video for all students in the network for learning or training the video offers a lot of information in a small amount of time and the video makes transmission behavior, emotion and education easier. The motivation of this paper is to broadcast the video over the network in a reliable manner by using the H.264 codec and controller to decrease bitrate with no effect on the quality. The video must be reliable to meet the link bandwidth and high quality for the consumers. additionally, find the fidelity of the protocols combination by QoS measurements. The rest of paper is expressed as follows, Section 2 represents routing and multicast protocols, Section 3 illustrates the video compression, Section 4 proposed model, while section 5 deal with the experiments and results and section 6 the conclusion of the proposed system.

\section{PROTOCOLS SPECIFICATION}

In a computer network, the transmission of data is based on the routing protocol which selects the best routes between nodes, routing protocols are classified into two types

- Static routing: it is built on the routing table manually and any change implemented by the administrator[4].

- Dynamic routing: - it allows the routers to select the best path according to the information that enters in the routing table continuously.

The dynamic routing divided into two types distance vector calculate the path cost according to hop account (number of routers) and link-state protocols use advanced algorithms that maintain the network database. Figure 1 shows the types of routing protocols. The Protocol Independent Multicast (PIM) provides support for routing multicast traffic between routers within an autonomous system. 


\section{International Advanced Research Journal in Science, Engineering and Technology}

Vol. 5, Issue 8, August 2018

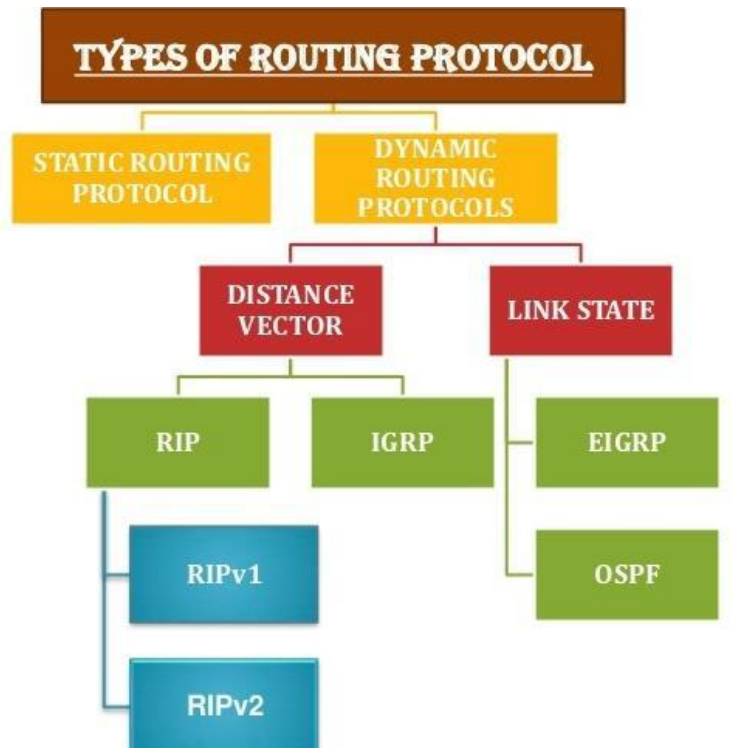

Figure-1 shows the routing protocols types

The most popular routing protocols are (RIP, OSPF, and EIGRP) dealing for routing the packets between the same network nodes, the OSPF protocol will be used as the routing protocol and Protocol Independent Multicast (PIM) to support the routing multicast traffic between routers within an autonomous system.

OSPF (Open Shortest Path First): Open Shortest Path First (OSPF) is a link-state routing protocol. It computes the shortest path tree for each route using a method based on the Dijkstra algorithm, a shortest path first algorithm. OSPF is used to determine the best route for delivering the packets within an IP network. It gathers link state information from available routers and constructs a topology map of the network. The topology determines the routing table presented to the Internet Layer which makes routing decisions based solely on the destination IP address found in IP packets, It is clearly faster routing protocol compared to previous protocols [5], the best path is chosen by the route with the lowest cost. The Cisco implementation of OSPF uses bandwidth to determine the cost. The path cost of an interface in OSPF is called metric that indicates standard value such as speed, the cost of an interface is calculated on the basis of bandwidth. High bandwidth indicates low cost[5].

IP Multicast Protocols: Is a method of sending Internet Protocol (IP) datagrams to a group of interested receivers in a single transmission, IP multicast uses the multicast distribution tree to describe the data transmission path in the network. There are two kinds of multicast distribution tree, including the source-based tree and shared tree. A sourcebased tree lies in the shortest path routing protocol, so also known as the shortest path tree (SPT), shared tree sends data from the multicast source to the public multicast router, then to the hosts of the multicast group[6]. PIM (Protocol Independent Multicast) has two modes it can function in, Dense Mode (DM) and Sparse Mode(SM). PIM-DM assumes that the members of a group are densely distributed in the network, and there is enough bandwidth in the network[7].

\section{THE VIDEO BROADCASTING OVER E-LEARNING CHALLENGES}

This section depicts the main E-learning requirements for broadcasting the video upon multi-access channels, shuch that of low bitrate, high quality, meet the resolution for the user terminals, and low delay in real time video. To such requirements there are challenges whic are; the bit rate of raw video is larger than transmission channel, to get low bitrate may there is loss in the quality of the video, and the nature of video is (VBR) and channel of (CBR) need an accurate buffering system designed to avoid buffer overflow and underflow for the encoder and decoder.

\section{VIDEO COMPRESSION}

The mentioned requirements and challenges are overcome by using an H.264 as the core codec of an optimal configuration. The system operation will explain the optimal usage of coder and decoder tools to make bit rate compatible with channel capabilities. The H.264 steps will demonstrate below:

Video formatting: - in this step will test the video formats that are desired for broadcasting. The video definitions like HD, CIF and QCIF have different dimensions as explained in figure 2. 


\section{International Advanced Research Journal in Science, Engineering and Technology}

Vol. 5, Issue 8, August 2018

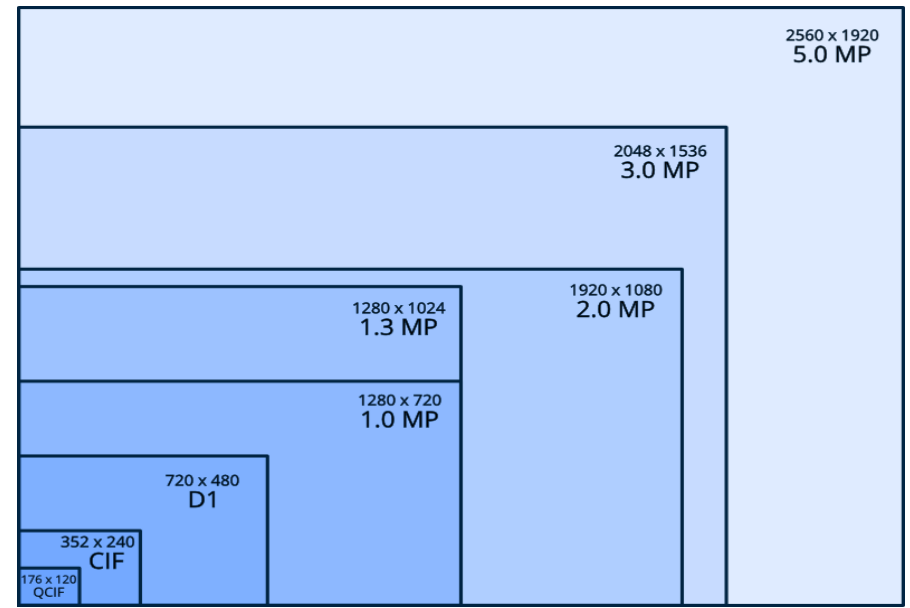

Figure-2 shows the video definition

Video encoding: - this step demonstrates the steps of the encoder, as in figure 3 depicts the video will enter the encoder as a sequence of frames for processing.

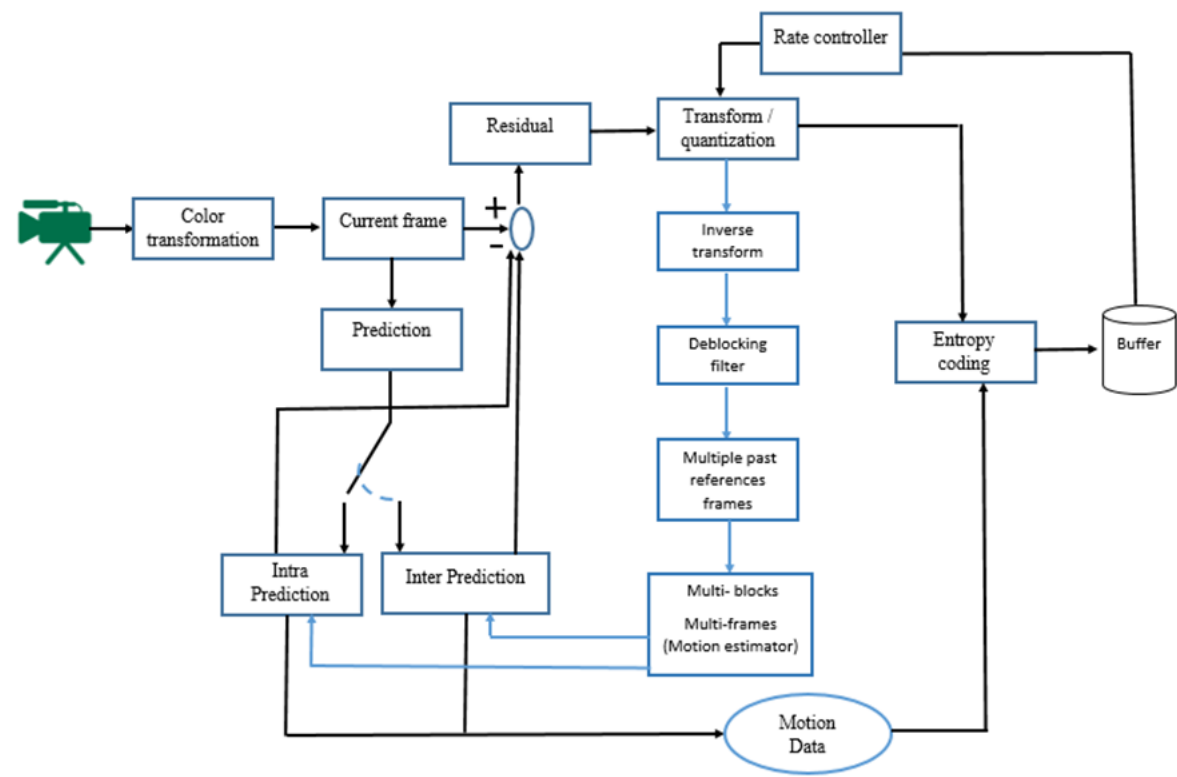

Figure-3 shows the H.264 encoder

The first step in the video coding transforms the video into YUV formatting then chose the down sampling for the video in this paper will choose the 4:2:0 as shown in figure 4

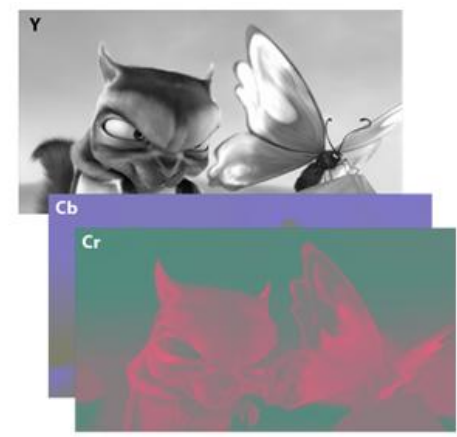

(a)

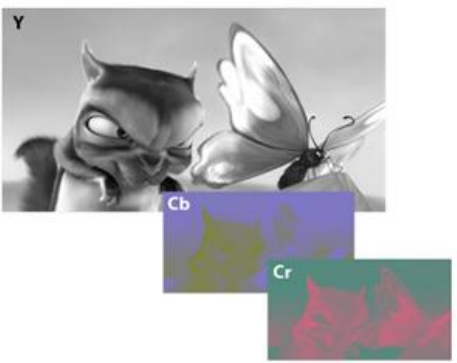

(b)

Figure-4 depicts the 4:2:0 down sampling 


\title{
International Advanced Research Journal in Science, Engineering and Technology
}

\author{
Vol. 5, Issue 8, August 2018
}

\section{The prediction step:}

The prediction step is divided into two parts intra prediction and inter prediction

The intra prediction: Prediction of the current block from the neighboring samples. The current frame will be coded without using a reference frame, it is based on the macro blocks in the current frame.

The inter prediction: Predicting the current MB from the previously encoded frame that is after or before the current frame according to the display order it has two processes motion estimation and motion compensation. The motion estimation is to choose the best match for two macro blocks, while the motion compensation is to is the process of subtracted the candidate blocks from the current block.

H.264 transformation: The residual data after motion compensation will be transformed by the DCT and quantized by a quantization parameter.

Ordering and entropy coding: The zigzag ordering is applied after the transformation and coding the ordering by the CABAC.

\section{The rate controller}

The rate control usually concerns about the specific problem, the buffer is placed between the video encoder and channel. The buffer has the limited capacity it is lead to delay when it is overflow or underflow, therefore the proposed rate controller responsible for regulating the coding parameters to achieve optimal video quality and ensure the buffer never underflows or overflows. The rate controller that is working for h.264 is consists of three techniques that are used to control the data rate as follows:

1. GOP rate control level: at the beginning of the ith GOP, the total number of bits allocated for the $\mathrm{I}^{\text {th }}$ GOP is computed as follow:-

$$
B_{i}(j)=\left\{\begin{array}{cl}
\frac{R_{i}(j)}{f} \times N_{i}-V_{i} & j=1 \\
B_{i}(j-1)+\frac{R_{i}(j)-R_{i-1}(j)}{f} \times\left(N_{i}-j+1\right)-b_{i}(j-1) & j=2,3, \ldots
\end{array}\right.
$$

Where $\mathrm{i}$ is the GOP number while $\mathrm{j}$ is the frame number in the $\mathrm{i}^{\text {th }}$ GOP while $\mathrm{f}$ is the frame rate and the $\mathrm{N}_{\mathrm{i}}$ the size of the $i^{\text {th }}$ GOP, while $R_{i}(j)$ the instant available bit rate, $B_{i}(j)$ actually generated bits and $V_{i}(j)$ is occupancy of the buffer for the $j^{\text {th }}$ picture in the $i^{\text {th }}$ GOP. The instant available bit rate may be variable for VBR while in the CBR the $R_{i}(j)$ is always equal to $\mathrm{Ri}(\mathrm{j}-1)$ and $\mathrm{b}_{\mathrm{i}}(\mathrm{j}-1)$ is the buffer occupancy for previous GOP.

2. Frame rate control level: in this step will compute the bit allocation for every frame in the GOP. The buffer occupancy is specified with its threshold for using the skip frame technique when the threshold has reached the frame that is used use very little macro blocks used will be skipped and using the next frame as a reference. It adjusts the buffer capacity. The rate controller usually adjusts buffer capacity that is occupied by using the skip frame technique, when occupancy reaches a certain threshold, so skip one or more frames tend to prevent buffer overflow.

3. Basic unit rate control step: processing of basic unit layer and the image layer is basically almost the same. And one frame can be divided into one or more basic units. The coding parameters that are used to influence the rate control are Quantization parameter (QP), Group of Picture (GOP), Skip frames, multiple references frames and MB partitioning, Main-partitioning and sub-partitioning, that rae detailed below as used in the proposed system.

Quantization parameter (QP): It controls the trade-off between compression efficiency and the frame quality.it is used for all the rate control levels to allocate the bits for every frame in the video. It is used to change the compression ratio in order to match the coded bit rate to a transmission channel rate. The quantization parameter QP can only influence the detail of information carried in the transformed residuals, the controller will guarantee the perceptible variation in the bitrate and the quality. So when the buffer arrives at the threshold area the QP will change noticeably, therefore, the encoder will send the variation in the QP to the decoder. The application of the quantization parameter is depicted below:

1. The frame divided into $4 * 4$ macro blocks.

2. Choose a quantization parameter value from the codebook that matches the current block as closely as possible.

3. Transmit an index that identifies the chosen vector to the decoder.

4. At the decoder, reconstruct an approximate copy of the region using the selected vector. 


\section{International Advanced Research Journal in Science, Engineering and Technology}

Vol. 5, Issue 8, August 2018

Figure 5 shows the quantization parameter mechanism.

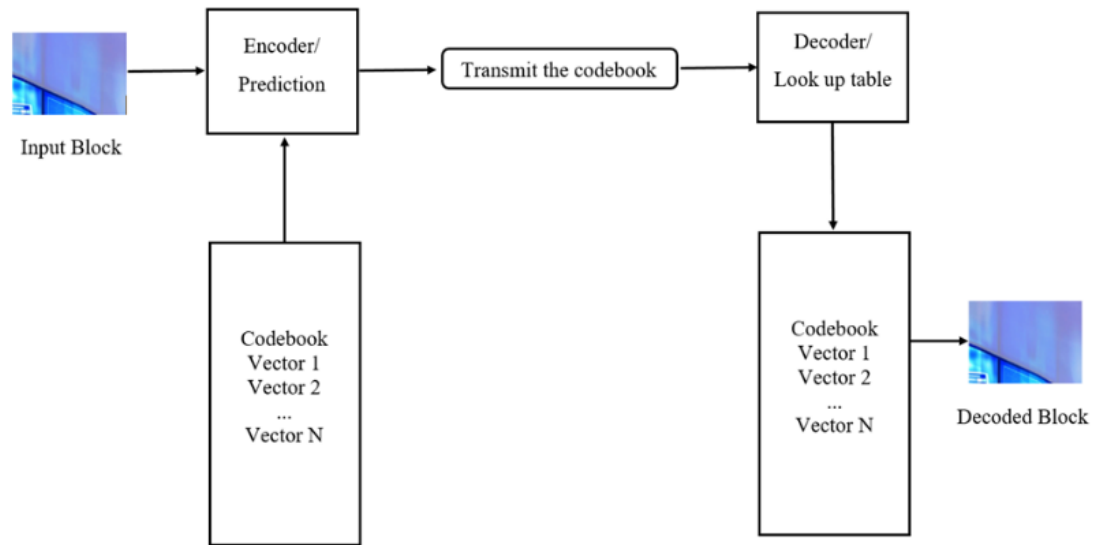

Figure-5 depicts the quantization parameter working

Group of Picture (GOP): The length of GOP means length between two sequential I-frames. Generally, reducing GOP length leads to reduction quality and a number of bits. The I frames have the high quality it constructed spatially (intra prediction) not depend on another frame. Typically, the encoder uses GOP structures that cause each I frame is new intra period.

Skip frames: The skip frame it is important parameter for the adjusted buffer threshold when the occupancy reach the certain threshold it must skip number of frame to prevent the buffer overflow. Figure 6 shows the rate controller working.

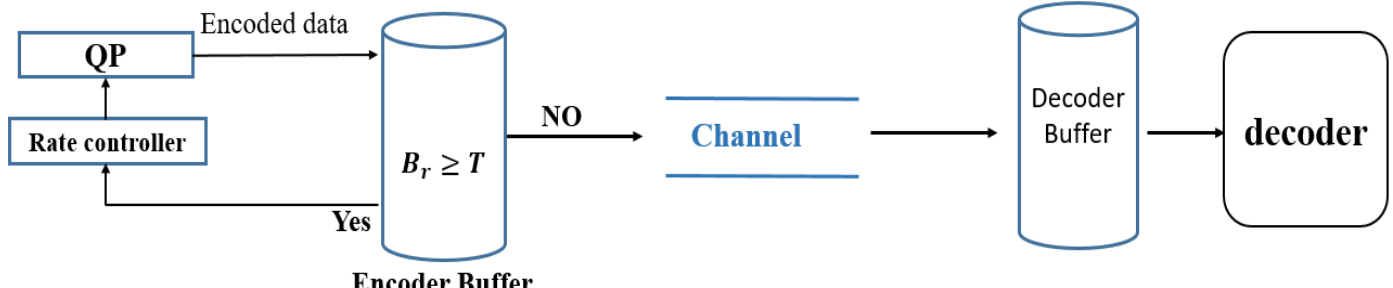

Figure-6 shows the proposed controller mechanism

Multiple references frames: This means that the encoder can search up to $\mathrm{N}$ reference frames to find the best match for each P macro block, this can improve compression efficiency. The skip frame, GOP, references frames and the buffer threshold are configured for the controller while the QP is dynamic parameter for adjusting the output bitrate to be compatible with buffer threshold and channel capacity.

\section{THE VIDEO BROADCASTING}

In this section, the E-learning system based on video broadcasting is modeled. To demonstrate the encoder-networkdecoder, the system shown in figure 7 is suggested as a media for testing and measurements. The network topology, devices that are used in the proposed network and the protocols in the proposed model will be explained.

The proposed model: The reliability of transmitting packets from the source to the destination is necessary. The problems that occur through broadcasting like the congestion and the link dropping are the most popular in the network, therefore, the network topology is designed to provide reliability overcomes the Mentioned problems. The server will transmit the packets for users with specific bitrate through limited links. So the router that is used in our topology is c7200 with exceptional performance, modularity, and scalability in a compact form factor with a wide range of deployment options. The hub transmits the packets for all end terminals shared in the network. The OSPF protocol is used to ensure the broadcasting for all end users while the PIM-DM is used to forward the packets and prevent the forwarding loop in the packets transmission. 


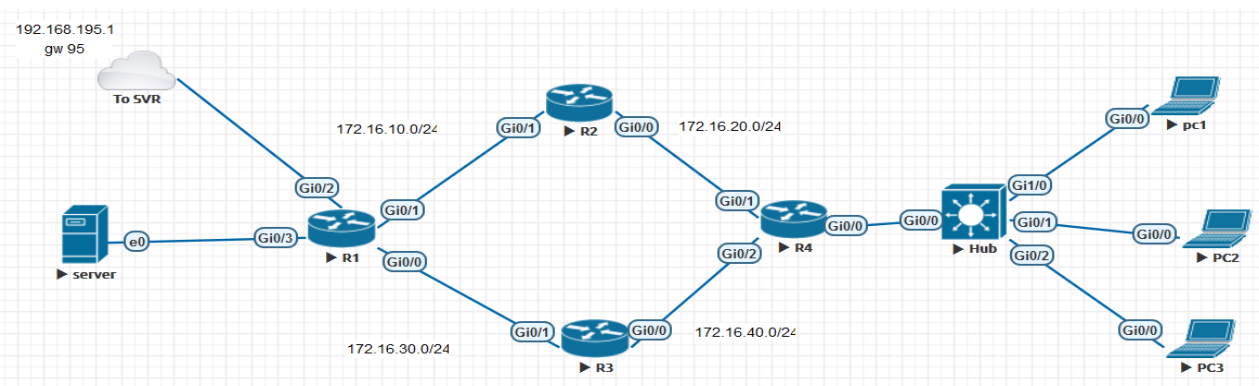

Figure-7 shows the proposed network

\section{THE EXPERIMENTS AND RESULTS}

This section demonstrates the results of the model and depicts the performance of optimal set of the controller parameter for making compatible with channel bandwidth for HD definition as example over main profile table 1 depicts different set when the setting the $\mathrm{GOP}=10$, skip frame $=4$, and references frames $=3$ with different values for QP.

Table-1 summarize the video

\begin{tabular}{|c|c|c|c|c|c|c|c|c|c|}
\hline QP & $\begin{array}{c}\text { Y } \\
\text { PSNR }\end{array}$ & $\begin{array}{c}\text { U } \\
\text { PSNR }\end{array}$ & $\begin{array}{c}\text { V } \\
\text { PSNR }\end{array}$ & $\begin{array}{c}\text { total bits } \\
\text { for I }\end{array}$ & $\begin{array}{c}\text { total bits } \\
\text { for P }\end{array}$ & $\begin{array}{c}\text { total } \\
\text { bits for } \\
\text { B }\end{array}$ & total bits & $\begin{array}{c}\text { bitrate } \\
\text { /Kbps }\end{array}$ & CR \\
\hline $\mathbf{2 0}$ & 44.38 & 51.27 & 51.18 & 2196056 & 5258792 & 557080 & 8012112 & 3697.9 & 403 \\
\hline $\mathbf{2 5}$ & 42.42 & 49.304 & 49.18 & 1526024 & 2964392 & 556552 & 5047152 & 2329.45 & 640 \\
\hline $\mathbf{2 8}$ & 41.1 & 48.16 & 48.1 & 1172968 & 1943984 & 560232 & 3677368 & 1697.25 & 879 \\
\hline $\mathbf{3 0}$ & 40.20 & 47.528 & 47.50 & 973160 & 1457480 & 575512 & 3006336 & 1387.54 & 107 \\
\hline
\end{tabular}

The optimal set for the controller when the QP $=28$ because the low bitrate with accepted quality for PSNR value and will be used as the default set in the controller. The performance of the proposed work for broadcasting in e-Learning the network with HD definition for bitrate $3697.9 \mathrm{Kbps}$ by measure the end-to-end delay, jitter, and finally, throughput, the performance of devices in the network is measured and explain the fidelity of the proposed model. Figure 8 shows the end-to-end delay for each device in the network.

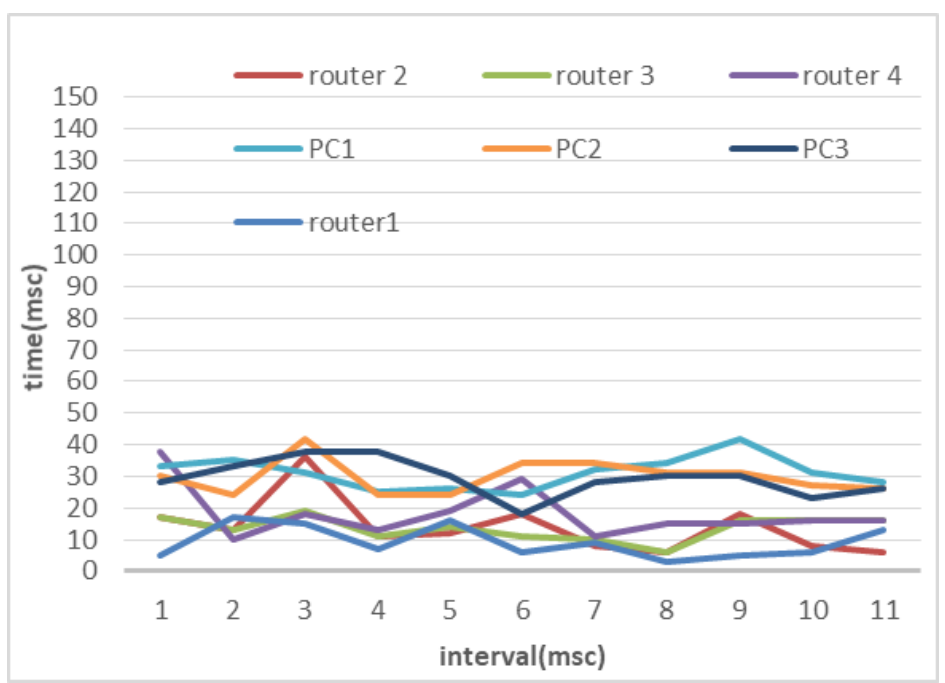

Figure-8 shows the end-to-end delay

So the end-to-end delay results explain that the packets are taking acceptable time for transmitting from one node to another, and notice is first moments the users take clearly delay for receiving the packets because of the number of the nodes in the transmitting media for the network, Table 1 depicts the facts. 


\section{International Advanced Research Journal in Science, Engineering and Technology}

Vol. 5, Issue 8, August 2018

Table 1 shows the end-to-end delay for overall network

\begin{tabular}{|c|c|c|c|c|c|c|c|}
\hline Time(MSC) & R 1 & R 2 & R 3 & R 4 & PC1 & PC 2 & PC3 \\
\hline $\mathbf{0}$ & 5 & 17 & 17 & 38 & 33 & 30 & 28 \\
\hline $\mathbf{2 0}$ & 17 & 13 & 13 & 10 & 35 & 24 & 33 \\
\hline $\mathbf{4 0}$ & 15 & 36 & 19 & 18 & 31 & 42 & 38 \\
\hline $\mathbf{6 0}$ & 7 & 11 & 11 & 13 & 25 & 24 & 38 \\
\hline $\mathbf{8 0}$ & 16 & 12 & 14 & 19 & 26 & 24 & 30 \\
\hline $\mathbf{1 0 0}$ & 6 & 18 & 11 & 29 & 24 & 34 & 18 \\
\hline $\mathbf{1 2 0}$ & 9 & 8 & 10 & 11 & 32 & 34 & 28 \\
\hline $\mathbf{1 4 0}$ & 3 & 6 & 6 & 15 & 34 & 31 & 30 \\
\hline $\mathbf{1 6 0}$ & 5 & 18 & 16 & 15 & 42 & 31 & 30 \\
\hline $\mathbf{1 8 0}$ & 6 & 8 & 16 & 16 & 31 & 27 & 23 \\
\hline $\mathbf{2 0 0}$ & 13 & 6 & 16 & 16 & 28 & 26 & 26 \\
\hline
\end{tabular}

In figure 9 the time of delivery between each packet and another for each device in the network is explained.

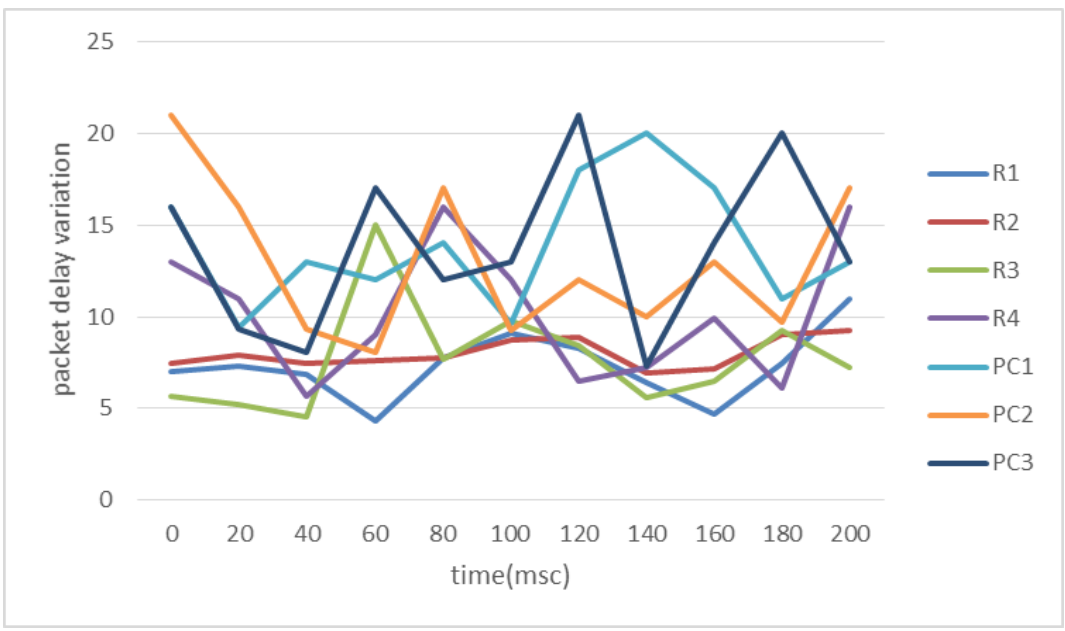

Figure-9 depicts the jitter for the packets

The jitter between the packets is acceptable and the variation between packets is tolerable for the users, the jitter from the hub to the users is clearly there is variation because the packets transmitted to the hub by the last router through two paths, therefore, cause variation in the transmitting between the users and the hub, table 2 explain the jitter for the whole network.

Table-2 summarizes the jitter in overall terminals

\begin{tabular}{|c|c|c|c|c|c|c|c|}
\hline Time(msc) & R 1 & R 2 & R 3 & R 4 & PC1 & PC 2 & PC3 \\
\hline $\mathbf{0}$ & 7 & 7.49 & 5.68 & 13 & 16 & 21 & 16 \\
\hline $\mathbf{2 0}$ & 7.29 & 7.93 & 5.2 & 11 & 9.44 & 16 & 9.33 \\
\hline $\mathbf{4 0}$ & 6.86 & 7.49 & 4.53 & 5.7 & 13 & 9.33 & 8.04 \\
\hline $\mathbf{6 0}$ & 4.34 & 7.63 & 15 & 9 & 12 & 8.04 & 17 \\
\hline $\mathbf{8 0}$ & 7.75 & 7.79 & 7.65 & 16 & 14 & 17 & 12 \\
\hline $\mathbf{1 0 0}$ & 9.11 & 8.71 & 9.76 & 12 & 9.66 & 9.25 & 13 \\
\hline $\mathbf{1 2 0}$ & 8.32 & 8.86 & 8.42 & 6.51 & 18 & 12 & 21 \\
\hline $\mathbf{1 4 0}$ & 6.38 & 6.94 & 5.58 & 7.25 & 20 & 10 & 7.31 \\
\hline $\mathbf{1 6 0}$ & 4.7 & 7.15 & 6.48 & 9.9 & 17 & 13 & 14 \\
\hline $\mathbf{1 8 0}$ & 7.43 & 9.06 & 9.23 & 6.15 & 11 & 9.73 & 20 \\
\hline $\mathbf{2 0 0}$ & 11 & 9.28 & 7.22 & 16 & 13 & 17 & 13 \\
\hline
\end{tabular}




\title{
International Advanced Research Journal in Science, Engineering and Technology
}

\author{
Vol. 5, Issue 8, August 2018
}

Figure 10 shows the transmission throughput for the transmitted packets through the limited links.

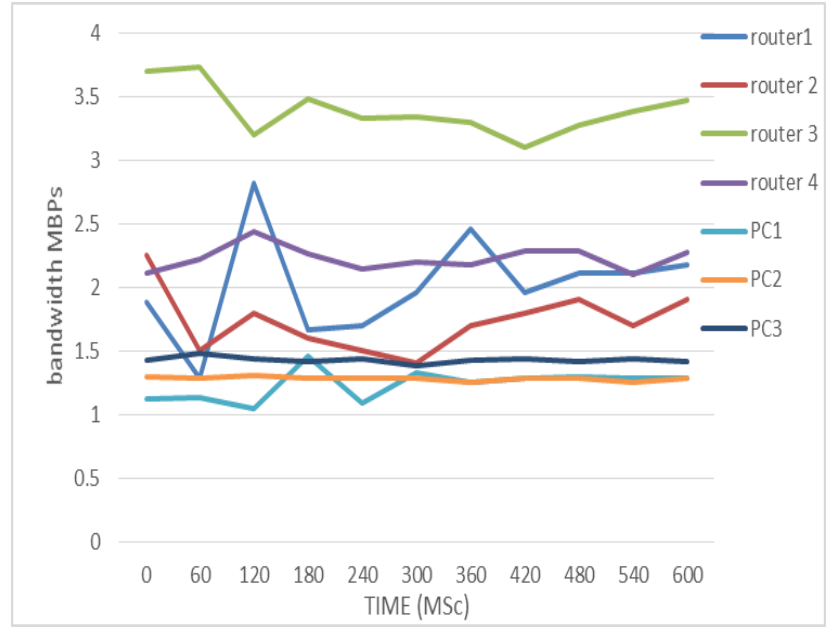

Figure-10 the transmission throughput of packets

The throughput of the transmitting and deliver the packets for the users and between the nodes of the network with limited links bandwidth with acceptable traffic, table 3 brief the bandwidth for the whole network.

Table-3 summarizes the transmission throughput

\begin{tabular}{|c|c|c|l|l|l|l|l|}
\hline Time(msc) & R 1 & R2 & R3 & R4 & PC1 & P2 & Pc3 \\
\hline 0 & 1.88 & 2.25 & 3.7 & 2.11 & 1.12 & 1.3 & 1.43 \\
\hline 20 & 1.29 & 1.5 & 3.73 & 2.22 & 1.13 & 1.28 & 1.48 \\
\hline 40 & 2.82 & 1.8 & 3.2 & 2.44 & 1.05 & 1.31 & 1.44 \\
\hline 60 & 1.67 & 1.6 & 3.48 & 2.26 & 1.46 & 1.29 & 1.42 \\
\hline 80 & 1.7 & 1.5 & 3.33 & 2.14 & 1.09 & 1.28 & 1.44 \\
\hline 100 & 1.96 & 1.4 & 3.34 & 2.2 & 1.33 & 1.29 & 1.38 \\
\hline 120 & 2.46 & 1.7 & 3.3 & 2.18 & 1.25 & 1.25 & 1.43 \\
\hline 140 & 1.96 & 1.8 & 3.1 & 2.28 & 1.29 & 1.28 & 1.44 \\
\hline 160 & 2.11 & 1.9 & 3.27 & 2.29 & 1.3 & 1.29 & 1.41 \\
\hline 180 & 2.11 & 1.7 & 3.38 & 2.1 & 1.28 & 1.25 & 1.44 \\
\hline 200 & 2.18 & 1.9 & 3.47 & 2.27 & 1.29 & 1.28 & 1.42 \\
\hline
\end{tabular}

The efficiency is achieved when using the combination of the OSPF and PIM-DM is clearly in the experiment results. So in the end-to-end delay, the path taken between the datagram's source and any consumer in the network is the least cost path available. The cost is expressed in terms of the OSPF link-state metric if the OSPF metric represents a delay, a minimum delay path is chosen. In the experiments results demonstrate the jitter plays an important factor in video streaming, it is explained more stability because the protocols combination (OSPF and PIM-DM) decrease forwarding packet time between devices. The throughput with simple variation notices it is decreased when the packets flooding is increased.

\section{CONCLUSION}

In this paper, the reliability is a very important factor, therefore must be achieved by using the H.264 codec. The using of the H.264 is enhances of the network performance and provide the reliability for the network users by ensure receiving the video by the users through avoiding the congestion and decrease the delay. The combination between OSPF and PIM-DM uses both source and destination to send the packets based on the information in the OSPF link state database about the system's topology. The PIM identify the users in the network then the shortest path calculated from that information by OSPF. 


\section{International Advanced Research Journal in Science, Engineering and Technology}

Vol. 5, Issue 8, August 2018

\section{REFERENCES}

[1]. S. Misra and S. Goswami, Network Routing: Fundamentals, Applications, and Emerging Technologies. 2017.

[2]. P. Browning, F. Tafa, D. Gheorghe, and D. Barinic, "Cisco CCNA Simplified your complete Guide to passing the Cisco routing and switching exam 5-Edition," Paul Browning, 2017.

[3]. P. L. Dordal, An Introduction to Computer Networks Principle : Layering Layering. 2018

[4]. C. Archana, “Analysis of RIPv2, OSPF, EIGRP Configuration on router Using CISCO Packet tracer,” Int. J. Eng. Sci. Innov. Technol., vol. 4, no. 2, pp. 215-222, 2015.

[5]. G. E. Clarke, Compatia Network+, Sixth Edit. McGraw-Hill Education, 2015.

[6]. Z. Li, L. Gao, and H. Ren, "IP multicast, theater monitoring video, video transmission, Winsock," 3rd Int. Conf. Mater. Mech. Manuf. Eng., no. Ic3me, pp. 820-824, 2015.

[7]. D. L. J., R. A. H., and . A. H. S., "Multicast Delivery of IPTV Over the Internet,” GSTF J. Comput., vol. 1, no. 1, pp. 164-169, 2014.

\section{BIOGRAPHY}

Mahmood Abdul Hakeem Abbood, born in April 1989 and graduated from Anbar University / computer college and programmer in ministry of science and technology/ information technology office, I have ICDL certificate and worked in the Iraq E-portal now study master in computer science in the Iraqi Commission for Computers and Information / Informatics Institute for Postgraduate Studies. 